\title{
ALGUNOS FACTORES QUE PUEDEN INFLUIR EN EL CRECIMIENTO DE LAS PEQUEÑAS Y MEDIANAS EMPRESAS TURÍSTICAS DEL SECTOR HOTELERO EN ACAPULCO-MÉXICO
}

\author{
SERGIO FRANCISCO REYNA PINEDA \\ Unidad Académica de Turismo. Universidad Autónoma de Guerrero. \\ Campus Acapulco, Guerrero, México. \\ sergioreyna@hotmail.com \\ DORA MA. OCAMPO HERRERA \\ Unidad Académica de Lenguas Extranjeras. Universidad Autónoma de Guerrero \\ Campus Acapulco, Guerrero, México. \\ dorisocampo@hotmail.com
}

\section{RESUMEN}

En México la actividad turística es fundamental para el desarrollo y la economía, siendo una importante generadora de empleos, divisas y derrama económica. Ante este entorno, quienes juegan un papel estratégico son las Pequeñas y Medianas Empresas Turísticas (Pymest), ya que representan un alto porcentaje en relación al total de las empresas turísticas, sobresaliendo el sector hotelero, participando en la creación de empleos, generación de ingresos y contribuye significativamente al bienestar económico y social de un destino turístico como México. El objetivo de este trabajo de investigación empírico, es identificar y analizar algunos factores que pueden influir en el crecimiento de las Pymest particularmente del sector hotelero de Acapulco-México, a partir de la percepción y opinión de los dueños/gerentes. Esta investigación se realizó en el periodo de primavera de 2009, se tomó en cuenta la población total de 176 miembros de la Asociación de Pequeños Hoteles y Moteles de Acapulco, se aplicó un cuestionario a los dueños/gerentes, utilizando una escala tipo Likert, respecto a los siguientes factores; a) atractivo de la actividad turística, b) características de la empresa y c) características empresariales del dueño/gerente. Los principales resultados fueron: 1) la mayoría de los dueños/ gerentes de las Pymest en Acapulco consideran al mercado turístico y a la imagen de la actividad turística muy bien posicionados, no así en el caso del producto turístico "Acapulco", pues con respecto a los niveles de percepción en cuanto a su atractivo, la mayoría no mostró una clara definición de su posición pues la calificaron como regular. Situación que genera incertidumbre a la hora de realizar nuevas inversiones en el sector turístico de Acapulco; 2) a pesar de lo anterior, se encontró que el promedio de ocupación anual de los hoteles participantes fue de $72 \%$ en los tres años previos a la aplicación del estudio, destacando que solo uno de estos hoteles recibe turistas internacionales mayoritariamente y el resto turistas nacionales; 3) la mayoría de los administradores de los hoteles participantes son los propios dueños de la empresa y/o sus familiares, situación que llama la atención debido a que en la mayoría de los casos no cuentan con antecedentes familiares empresariales. En cuanto al conocimiento que los dueños/gerentes de las Pymest poseen sobre la actividad turística en general, se encontró que menos de la mitad manifiestan tener un amplio conocimiento del tema, sin embargo, en lo relativo a su experiencia en la hotelería, el 73\% indicó que su experiencia particular es amplia.

PALABRAS CLAVES: PYMEST, CRECIMIENTO, ORIENTACIÓN EMPRESARIAL, DESARROLLO EMPRESARIAL, ACTIVIDAD TURÍSTICA. 


\section{ABSTRACT}

In Mexico the tourist industry is core to its economy because it generates jobs, foreign exchange and significant economic spill. With this background those who play a strategic role, are the Small and Medium Tourism Enterprises (SMET's) as they represent a large percentage of the total number of tourism businesses being most important within the hotel sector, due to its participation in job and income generation and significant contribution to economic and social welfare as a tourist destination. The objective of this research is to identify and to analyze factors that might contribute to growth of the hospitality industry SMET's in Acapulco-México, based on owner/manager's perception and opinion. This research conducted in spring 2009, took into account the total population of 176 members of Small Hotels and Motels Association in Acapulco, applying a questionnaire to the owners/managers using a Likert scale, concerning factors such as a) tourist industry attractiveness, b) hotel features and c) owner/manager's entrepreneurial features. In this paper we present some advances of the results. Main contributions of this study can be summarized as follows: 1) Most of the owners/managers of SMET's in Acapulco-Mexico still consider the Tourism Market as well as the Image of the Industry, in a high positive position, whereas the perception of "Acapulco" as a Touristic Product shows an uncertain position regarding its attractiveness; 2) despite the former situation, the average year occupancy in participant hotels was 72\% during the three previous years of this study, noticing that only one of these hotels was hosting mainly foreign tourism, and the rest of them received domestic tourism; 3 ) it was found that most of the SMET's administrators were owners of the hotel or relatives of the owners. This situation becomes relevant since they did not have entrepreneurial background. Regarding previous knowledge of the tourism activity, owners/managers of SMET's expressed low understanding of the area, however, when referring to hotel experience, $73 \%$ declared having wide experience in this topic.

KEY WORDS: SMET'S, GROWTH, ENTREPRENEURIAL ORIENTATION, ENTREPRENEURIAL DEVELOPMENT, TOURISM INDUSTRY.

\section{INTRODUCCIÓN}

La demanda de turismo mundial sigue superando expectativas, mostrando una resistencia ante factores externos que se podría decir desafían la lógica tradicional. Esto se refleja, por un lado, en que la demanda turística es el aspecto del sector turístico que más ha evolucionado en el mundo, tendiendo hacia una mayor diversificación de sus motivaciones para viajar, mayores exigencias de calidad del medio ambiente, de la prestación de servicios y hacia un turismo más personalizado, menos masificado y, por lo tanto, más activo. Por otro lado, la oferta turística se ha comportado de un modo más estático, como es el caso de las Pequeñas y Medianas Empresas Turísticas (Pymest), y solo en los últimos años han comenzado a reaccionar lentamente para adaptarse a esas nuevas exigencias de la demanda (OMT, 2008).

Las Pymest son organizaciones que conforman la columna vertebral de la economía de muchos países con una actividad turística importante, por lo tanto, deberían convertirse en el futuro en pilares de esa actividad en países con vocación turística (OMT, 2008).

México, país turístico por excelencia, no está ajeno a esta situación, es considerado como el octavo país del mundo receptor de turistas, contando con una gran diversidad de atractivos turísticos tanto naturales como culturales. Un ejemplo de ello es que en el año 2008 recibió 21,500 millones de turistas, logrando ingresos por concepto de divisas de 10 389,20 millones de dólares en ese mismo año (Sectur, 2009). Al mismo tiempo, la ciudad y puerto de Acapulco es considerado uno de los destinos de playa más importantes de México, donde casi el 90\% de los negocios son considerados Pymes (Sefotur, 2009). Siendo esta la razón fundamental para realizar esta investigación. 


\section{Antecedentes sobre las Pequeñas y medianas empresas (Pymes)}

En las últimas décadas se han realizado muchos estudios sobre las Pequeñas y Medianas Empresas, mostrando que son una fuente importante en la creación de empleo y riqueza (Drucker, 1985; Birch, 1987; Storey, 1994). Sin embargo, a pesar del gran aumento en la investigación en esta área, los estudios existentes están lejos de generar un consenso a nivel teórico (Davidson y Wiklund, 1999) y mucho menos se ha generado un solo marco teórico e integrador para analizar el crecimiento de las empresas especialmente en el área del turismo. Sin embargo, el conocimiento de por qué algunas empresas muestran niveles de crecimiento, mientras que otras fracasan, es todavía insuficiente (Wiklund, 1998). Por otro lado, existe una gran convicción por parte de muchos investigadores de que incrementar los estudios del crecimiento de las Pymes es un tema clave para el desarrollo económico de cualquier país o región. De acuerdo con Turok (1991) existe un interés considerable en identificar las características principales que distinguen a las empresas que crecen, de aquellas más estables o aquellas que fracasan y desaparecen. Por un lado, es interesante conocer más sobre los procesos de crecimiento y desarrollo de las empresas e identificar los factores que afectan el crecimiento, por lo tanto, es un tema relevante para el diseño y aplicación de políticas de apoyo más selectivas por parte de empresarios y autoridades gubernamentales.

En este escenario, el mayor problema no es la falta de investigaciones sobre el crecimiento de las Pymes, sino que el campo carece de estudios conceptualmente fuertes y empíricamente comprensivos (Wiklund, 1998). De esta forma, aunque una gran cantidad de trabajos se han dedicado a identificar los factores de éxito en el crecimiento de las empresas, los distintos autores llegan a resultados bastante diferentes. Según Schutjens y Wever (2000) la principal ra- zón para estas discrepancias puede deberse a diferencias en el objetivo de la investigación ya que algunos trabajos analizan ciertos sectores específicos o empresas que difieren en sus actividades, mientras que otros consideran un amplio rango de factores y escenarios.

En la actualidad no existe una sola teoría que pueda explicar y medir adecuadamente el crecimiento de las Pymes y tampoco se perfila una teoría que pueda ser desarrollada en un futuro cercano. Esto se debe en parte, a la heterogeneidad que existe entre las Pymes y a la diversidad en los tipos de actividades al que se dedican, pero también al rango de factores que pueden afectar el crecimiento, los cuales pueden interactuar entre sí en diferentes formas y circunstancias. Aunque es posible identificar factores de éxito que afectan el crecimiento de las Pymes, es improbable que surja un solo modelo teórico comprensible con capacidad predictora que explique adecuadamente ese crecimiento (Smallbone, Leigh y North, 1995).

\section{Problemas recurrentes en el crecimiento de las Pequeñas y medianas empresas turísticas (Pymest)}

Durante la revisión de literatura se encontró que entre los problemas de mayor incidencia que enfrentan particularmente las Pymest se ubican los siguientes:

1. La incapacidad del dueño/gerente para manejar el crecimiento. Entendida como la habilidad para dirigir la organización tanto interna como externa en respuesta al crecimiento de su empresa, así como la habilidad para descubrir y explotar nuevas oportunidades de crecimiento (Covin y Slevin, 1989).

2. Los cambios en el entorno. Estos cambios (riesgos, tecnología, entre otros) crean diná- 
micas en la demanda y pueden abrir nuevas oportunidades de crecimiento para las empresas, pero si el dueño/gerente no los identifica y no los aprovecha, pierde la oportunidad de que su empresa crezca (Haber y Reichel, 2007).

3. La escasez de investigaciones sobre las Pymest. Como tendencia general, los enfoques de las investigaciones en el área -que son pocas-, comúnmente se limitan a propuestas de soluciones para "problemas externos" a la empresa, por ejemplo, mediante programas de apoyo a la industria o a simples especulaciones empíricas sobre sus "problemas internos de gestión"; en ambos casos las propuestas carecen de la validación cuantitativa o estadística, que es fundamental en estudios de carácter científico (Palomo, 2007).

4. La falta de información teórica y empírica en las Pymest, debido principalmente al alto costo de generar información estratégica para este tipo de empresas. Es importante mencionar que los únicos marcos de referencia para la mayoría de los estudios en este sector son los estudios regionales comúnmente llevados a cabo por los gobiernos con una perspectiva muy general (Weiermair, 2001).

Los aspectos anteriores nos indican que el conocimiento sobre las Pymest está en la etapa de generación de la información y la mayor parte del desarrollo conceptual en esta área se ha derivado de las investigaciones sobre las Pymes en general, dejando fuera las características propias de la actividad turística (Jogaratnan, Mclearly y Mena, 2005).

\section{Algunos factores que influyen en el crecimiento de las Pequeñas y medianas empresas turísticas (Pymest)}

Algunos de los factores que influyen en el crecimiento de las Pymest, se identifican en los trabajos de Haber y Reichel (2005, 2007); Monfort (2000); Capelleras y Kantis (2009); Wiklund y Shepherd (2003), Claver, Andrew y Quer (2006), Haktanir y Harris (2005), Chen (2009), Ateljevic (2007), entre otros. Entre estos factores se encuentra la aspiración de crecimiento del empresario, la edad y tamaño de la empresa, el atractivo de la actividad turística, los servicios que ofrece la empresa, la participación del gobierno, la orientación empresarial y habilidades empresariales, por mencionar algunos. De estos factores, los considerados de mayor relevancia para esta investigación fueron los siguientes:

\section{El entorno (atractivo de la actividad turística)}

El éxito de una empresa en cuanto a su desempeño y crecimiento depende de un gran número de factores relacionados a la organización y su entorno. El reconocimiento de esta complejidad ha obligado a muchos investigadores a incorporar en sus estudios factores como las características personales y empresariales del administrador, las organizacionales, el entorno, la orientación empresarial, las competencias de la empresa, la participación del gobierno, entre otros. La importancia del entorno para el desempeño de las empresas ha sido un aspecto reconocido por muchos investigadores y en especial en la actividad turística, en virtud de que el principal atractivo de muchas empresas turísticas está sustentada en los recursos y factores del entorno, lo que generalmente se denomina "el atractivo de la actividad turística" (Haber y Reichel, 2007). 
En este sentido, tomar vacaciones representa una actividad de ocio que involucra la interacción del entorno y sus componentes. Por lo tanto, los atractivos y los destinos turísticos por lo regular se planean sobre la base de componentes del entorno que pueden ser: geográficos, históricos, culturales, eventos, escenarios naturales, étnicos, entre otros (Milly y Morrison, 1992). Cabe mencionar que el entorno de la actividad turística incluye la acción conjunta de varios grupos de factores (económicos, sociales, culturales, políticos y otros) que influyen sobre el desarrollo de la actividad empresarial. Por esto, los componentes del entorno más próximo a la empresa, como la localización geográfica y la estructura del sector industrial, cobran especial relevancia en el análisis del crecimiento de las Pymes turísticas, ya que en estos componentes se refleja lo atractivo del mercado turístico, la oportunidad para el desarrollo de nuevos productos turísticos, el desarrollo empresarial y la oportunidad para nuevas inversiones en este sector (Haber y Reichel, 2007; Capelleras y Kantis, 2009).

\section{La empresa (características)}

El crecimiento de las empresas por lo general está relacionado con la competitividad de una industria de diferentes formas, por lo que muchos autores se enfocan en los recursos internos de las Pymes, como una variable explicativa para su crecimiento; las competencias medulares (core competencies) y el nivel de satisfacción del empresario con su profesión y su empresa son una de las variables que más influyen en el crecimiento de las Pymes (Pechlaner \& Matthias, 2004). En este sentido, el crecimiento continuo de una Pyme requiere la gestión y el desarrollo de diferentes competencias y capacidades, y de esta forma, el punto de vista interno de la empresa analiza el alineamiento de las estrategias de negocios con los recursos de la empresa.
A su vez, la búsqueda de competitividad de las Pymes por parte de los empresarios depende en gran medida de su permanencia en el mercado y de la capacidad de afrontar los nuevos retos empresariales, superando los sistemas de gestión tradicionales y adoptando un enfoque gerencial y de visión a futuro. Por su parte, Monfort (2000) menciona que la competitividad, el desempeño y el crecimiento de la industria hotelera se sustentan principalmente en los activos intangibles. Del mismo modo, los recursos físicos (ubicación, edificio, habitaciones, mobiliario) son fácilmente imitables por los competidores, debido a que son observados directamente y es posible su adquisición en el mercado. En cambio, los activos intangibles como la reputación de los hoteles, la imagen de la empresa, la calidad de los servicios de los empleados, las rutinas organizacionales de alojamiento, los servicios de reservaciones efectivos, no son fácilmente replicables dada la información imperfecta sobre sus factores determinantes $y$, adicionalmente, no se pueden comprar en el mercado.

\section{La orientación empresarial del dueño/gerente}

Al capital humano se le atribuye el nivel de desempeño de la empresa, esto se basa principalmente en la educación, experiencia y habilidades del empresario, y en las características empresariales de sus empleados. Algunos estudios han mostrado que efectivamente existe una relación positiva entre el nivel educativo y el desempeño empresarial, especialmente en sectores especializados como la industria turística (Cooper y Gimeno-Gazcon, 1994). Por otro lado, esos mismos autores encontraron que el knowHow específico y la semejanza entre negocios contribuyeron a la supervivencia y crecimiento de algunas empresas. Mientras que Miner (1996) menciona que la experiencia empresarial basada en factores como las características personales y antecedentes empresariales familiares pueden contribuir al éxito de una empresa. 
Desde esa perspectiva, la literatura sobre la estrategia y el entrepreneurship sugieren que la orientación empresarial mejora el desempeño de las empresas. La orientación empresarial involucra la voluntad de innovación, tomar riesgos, probar productos, servicios y mercados nuevos e inciertos, y ser consecuentemente más proactivos que los competidores hacia las oportunidades de nuevos mercados (Covin y Slevin, 1991). Por su parte, Wiklund y Shepherd (2003) en sus investigaciones sobre el desempeño y crecimiento en las Pymes, sugieren que la orientación empresarial reflejada en la innovación, la productividad y la acción de tomar riesgos en entornos dinámicos influye positivamente en el desempeño y crecimiento de las Pymes y que sería un error medir ese dinamismo y crecimiento basados en una sola variable.

\section{Las Pymest en México}

En lo que respecta a México, las Pymest representan un gran sector importante de las empresas turísticas establecidas, específicamente en el sector hotelero. Sin embargo, en la actualidad no se han realizado investigaciones con profundidad que nos indiquen con certeza ¿cómo se crean?, ¿cómo crecen?, ¿cuál ha sido su desarrollo? y ¿bajo qué condiciones cierran o quiebran? Se ha notado intensamente que las Pymest presentan grandes rezagos respecto a los sistemas de gestión utilizados por las grandes empresas cuyo desempeño está asociado a procesos modernos de gestión y personal altamente capacitado. La gestión de estas empresas (Pymest) está dirigida principalmente a la supervivencia, preocupadas por ir resolviendo los problemas que se les presentan a diario y generalmente muchos dueños/ gerentes aprenden de forma empírica.

Asimismo, en México no existe una verdadera actividad de investigación sobre las Pymest, y las investigaciones existentes solo describen en términos muy generales sus características y su papel en la economía. Por otro lado, las Pequeñas y Medianas Empresas (Pymes) en general, ya sea bajo la forma de empresa familiar o como empresa formalmente establecida, constituyen la principal fuente de generación de empleos, ya que representan más del $97 \%$ de los establecimientos que aportan cerca del 50\% de los empleos, según datos del Instituto Nacional de Estadística y Geografía e Informática (2009).

De acuerdo con la Secretaría de Turismo Federal con datos del año 2009, las Pymest conforman el $80 \%$ de la estructura del sector turístico en México, de las cuales, el sector hotelero representa casi el 90\%. De ahí la gran importancia que tienen estas empresas en México. Sin embargo, la gran mayoría enfrenta grandes problemas de supervivencia, requieren apoyo y orientación específica de acuerdo con sus necesidades para poder así mejorar sus instalaciones, diversificar sus productos y convertirse en organizaciones turísticas competitivas, y lo más importante, crecer y desarrollarse.

\section{METODOLOGÍA}

Características de la investigación

El estudio se presenta bajo un enfoque de investigación de tipo exploratoria, desde una perspectiva cuantitativa, descriptiva y transversal, es decir, la obtención de los datos se realizó en un solo momento, teniendo un carácter único en el tiempo. Los participantes fueron los dueños/gerentes de las Pymest del sector hotelero en Acapulco, quienes por su posición disponen de información relevante y de un amplio conocimiento de los recursos y capacidades que tienen sus empresas. Fueron consideradas las 176 Pymest integrantes de la Asociación de Pequeños Hoteles y Moteles de Acapulco-México. Para la obtención de la información se aplicó un instrumento de medición representado por un cuestionario aplicado exclusivamente a los dueños/gerentes de cada hotel. 


\section{Procedimiento}

Previo a la aplicación del instrumento de medición a los dueños/gerentes de los hoteles se estableció un plan de acción para lograr la aceptación de los empresarios, ya que al inicio se encontró un poco de resistencia para dar la información. Las acciones incluyeron los siguientes puntos: primero, se les explicó ampliamente la naturaleza y objetivos del trabajo de investigación y de la importancia de la información que ellos facilitarían; segundo, se les dio asesoramiento sobre el significado de las variables y cómo debían contestar el cuestionario; y tercero, se implementó un programa de concientización y convencimiento sobre la relevancia de sus empresas en la actividad turística. Como resultado de lo anterior, del total de los 176 hoteles considerados, respondieron completamente 132, lo cual representó una tasa de respuesta del 75\%, situación que resulta muy favorable para los objetivos de esta investigación. Para el análisis de la información recabada en los cuestionarios, se utilizó el programa SPSS 17,0 utilizando estadísticos descriptivos, frecuencias y tablas de contingencia. Por otro lado, la etapa desde la aplicación del plan de acción hasta la recepción de los cuestionarios tuvo una duración aproximada de 5 meses, marzo a julio de 2009.

Para fines de este trabajo, se tomaron en cuenta algunos de los factores identificados en la revisión de la literatura y considerados como los más relevantes para el objetivo de esta investigación: 1) el entorno (atractivo de la actividad turística), 2) la empresa (características) y 3) la orientación empresarial del dueño/gerente. A su vez, estos factores se analizaron a través de diversas variables -las cuales más adelante se explican-. Para la evaluación de las variables relacionadas con los factores entorno y orientación empresarial, se consideraron las opiniones personales de cada uno de los gerentes/dueños de los hoteles, obtenidas a través de los cuestionarios.
Variables del estudio

Para el factor Entorno "Atractivo de la actividad turística", se tomaron en cuenta cinco variables utilizadas en estudios previos por Bates (2005), Haber y Reichel (2005, 2007), Wiklund y Shepherd (2003), Dobbs y Hamilton (2007), Wiklund, Davidson y Delmar (2003), Pechlander, Raich, Zehrer y Peters (2004), siendo éstas: 1) atractivo del mercado turístico, 2) atractivo del producto turístico "Acapulco", 3) imagen de la actividad turística, 4) desarrollo empresarial y 5) oportunidad para nuevas inversiones. Para la medición de estas variables se utilizó una escala Likert con cinco ítems que fueron desde muy mala hasta muy buena.

Para el segundo factor denominado La Empresa "Características" se tomaron en cuenta seis variables: 1) número de habitaciones, 2) número de empleados trabajando, 3) número de años operando, 4) nivel de ocupación habitacional promedio anual en los años 2006, 2007, 2008, 5) tipo de turistas que recibe hotel mayoritariamente y 6) estancia promedio anual en días de sus huéspedes en los años 2006, 2007, 2008. Estas variables han sido utilizadas por autores como: Ateljevic (2007), Smith (2006), Rabetino (2005), Bates (2005), Phillips (1999), entre otros, quienes las han considerado fuente importante de información sobre el crecimiento del las Pymest.

Finalmente para el factor "Características empresariales del dueño/gerente", se consideraron nueve variables, cuatro de información básica como: 1) quién administra el hotel, 2) nivel educativo alcanzado, 3) sexo y 4) edad; y el resto enfocadas a las orientación empresarial del administrador como: 5) conocimiento de la actividad turística, 6) experiencia en la hotelería, 7) habilidades empresariales, 8) formación profesional y, 9) antecedentes familiares en el negocio. Para la medición de estas últimas se utilizó una escala Likert con cinco ítems que fueron desde muy 
débil hasta muy fuerte. Autores como Monfort (2002), Haber y Reichel (2007), Rubio y Aragón (2008), Davidson (2002), Mazzarol (2003), Calvo y López (2004) y Wiklund y Shepherd (2005), entre otros, han utilizado estas variables para explicar el crecimiento de las Pymest.

\section{RESULTADOS}

Tomando en cuenta la primera dimensión "atractivo de la actividad turística", los resultados muestran que del total de los dueños/gerentes entrevistados 28 perciben que el mercado turístico es muy bueno, 50 indican que es bueno y 38 que es regular, estos datos representan el 21\%, el $38 \%$ y el $29 \%$ del total general de esta variable. Si bien hay un 29\% de los entrevistados que perciben al mercado turístico como regular, casi el 59\% la consideran entre bueno y muy bueno. Lo anterior indica que la actividad turística en Acapulco aún tiene mucho que ofrecer y va en coherencia con que el turismo en México sigue siendo la actividad económica principal. En contraste, el 12\% del total de esta categoría la consideran entre mala y muy mala, lo cual indica que este segmento de gerentes considera que la actividad turística no tiene las condiciones para crecer en ese momento (ver figura 1).

FIgURA 1

\section{Atractivo del mercado turistico}

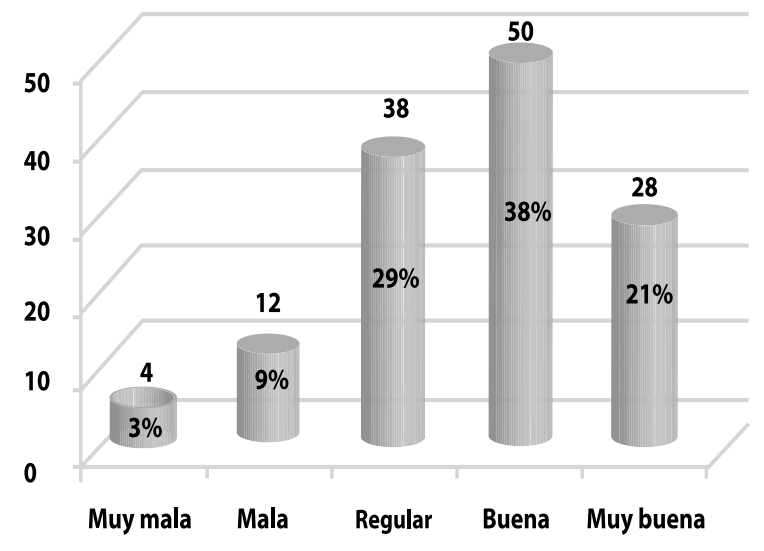

En la figura 2, se aprecian los resultados de la variable "Atractivo del producto turístico Acapulco", los cuales muestran que la percepción y opinión de los gerentes entrevistados no es definitiva de la posición de Acapulco como producto turístico, pues 51 de ellos, es decir, el 39\% consideran su posición como regular, mientras que 43 gerentes dijeron que Acapulco se encuentra entre una posición buena y muy buena, lo que representa solo el 32\% del total. Seguido de 38 gerentes que consideraron que este destino turístico está en una situación mala y muy mala, representando casi el $29 \%$ del total de esta categoría. Esto indica que, a pesar de no haber una clara tendencia de su posicionamiento como producto turístico, el puerto de Acapulco se mantiene como un destino turístico de playa con potencial.

\section{FIGURA 2}

\section{Atractivo del producto turistico "Acapulco"}

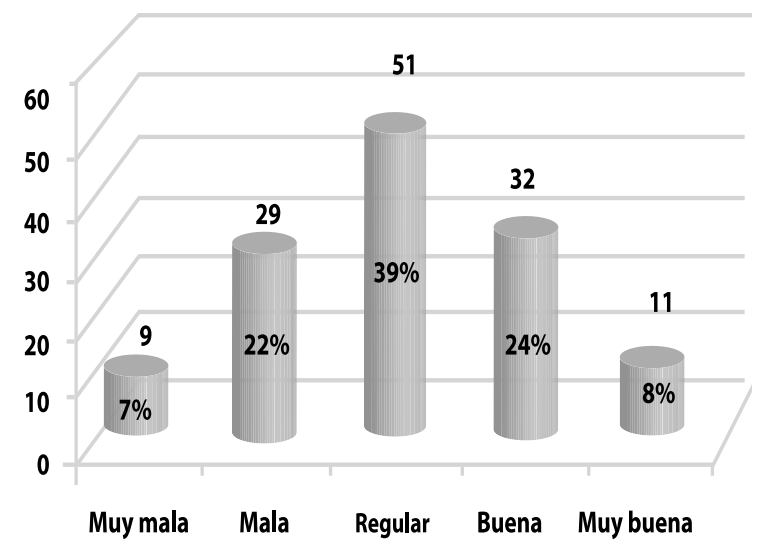

Sobre la variable "Oportunidad de desarrollo de nuevos productos turísticos", los resultados reflejan que 83 gerentes perciben que la situación se encuentra entre regular y muy buena, representando un 63\% del total, aunque 49 gerentes que representa el 37\% consideran que la situación se encuentra entre mala y muy mala, lo cual indica claramente que hay un buen porcentaje que pudiera aprovechar la oportunidad para desarrollar nuevos productos turísticos en su empresa sin 
que requiera mayor inversión, ya que se trata de crear nuevos productos turísticos innovadores y creativos para aumentar la estancia promedio de los turistas.

En lo referente a la variable "Imagen de la actividad turística", los resultados muestran que 80 gerentes del total de los entrevistados consideran la imagen de la actividad turística como buena y muy buena, esto representa el 60\%, los gerentes que consideran la imagen de la actividad turística como regular son 23, representando el 18\% del total en esta categoría (ver figura 3).

Figura 3

\section{Imagen de la actividad turistica}

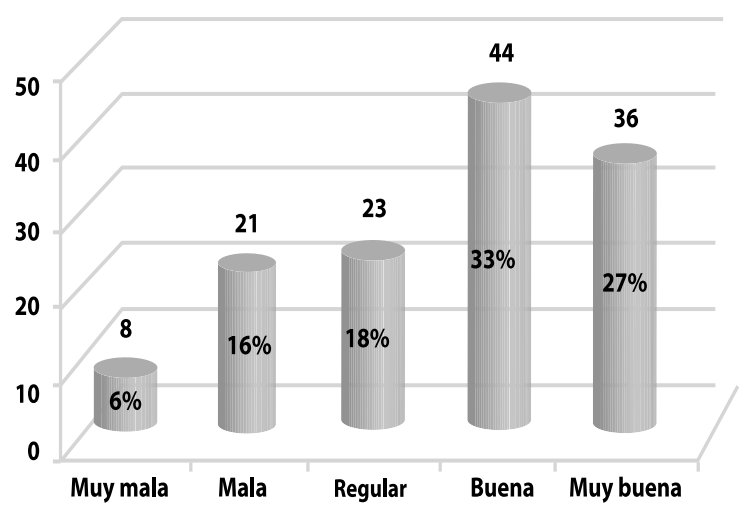

Finalmente sobre la variable "Desarrollo empresarial", los resultados muestran que aquellos que perciben la actividad turística como buena y muy buena para el desarrollo empresarial son 66 gerentes que representan el 50\% del total. Aquellos gerentes que perciben esta variable como regular son 52 y representa el 39\% del total, y los que consideran que no es buen momento para el desarrollo empresarial son 14 gerentes, representando el $11 \%$ del total de esta categoría.

Sobre la variable "Oportunidad para nuevas inversiones", del total de los entrevistados solo 40 consideran como buena y muy buena la oportunidad, esto representa el 30\%, aquellos que consideran regular la oportunidad representan 36 gerentes o sea el 27\% y significativamente, aquellos que la consideran mala representa 47 gerentes o sea el 36\% del total en esta categoría (ver figura 4). Lo anterior indica que son más los gerentes que perciben como mal momento para realizar nuevas inversiones (a nivel de las Pymest) a pesar de que la actividad turística es favorable para el destino turístico Acapulco.

\section{FIGURA 4}

\section{Oportunidad para nuevas inversiones}

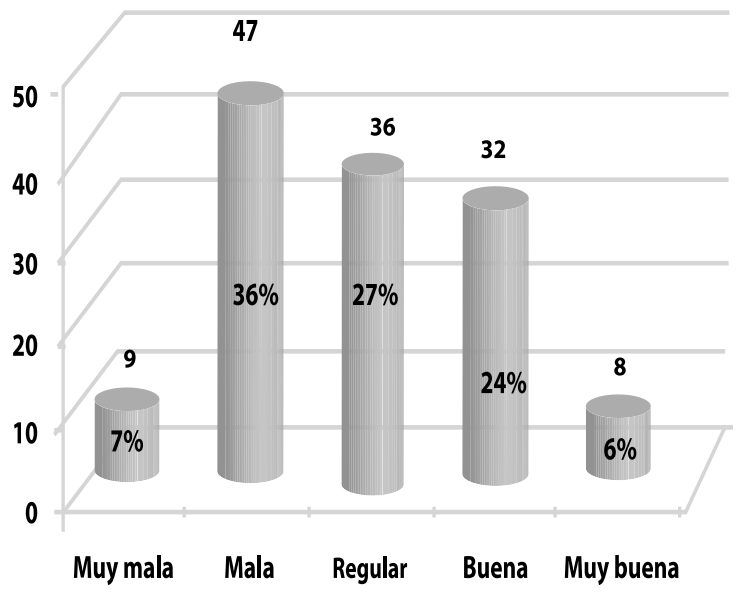

Los resultados de la segunda dimensión "Características de la Empresa", del nivel Identificación del hotel, se aprecian en la figura 5 y muestran los siguientes datos: del total de los dueños/gerentes entrevistados, 73 dueños administran su propio hotel, lo que representa un 56\%; familiares de algunos dueños administran 10 hoteles, lo que representa un 8\%; y 49 gerentes que no tienen ninguna relación familiar con el dueño administran algún hotel, lo que representa un 37\% del total. Lo anterior confirma el hecho de que casi la mayoría de las Pymes son administradas por los dueños. Sobre el nivel educativo alcanzado por quien administra un hotel, los resultados muestran que 50 gerentes tienen estudios máximos de bachillerato lo que representa el 38\% del total. Asimismo, 45 gerentes tienen estudios 
máximos de licenciatura que representa el 34\%, esto significa que predominan los gerentes que han alcanzado un nivel educativo de bachillerato. Agregando que 27 gerentes han alcanzado el nivel de secundaria, lo que representa el $21 \%$ del total. Finalmente, tres gerentes tienen estudios máximos de primaria y siete tienen el grado de posgrado, lo que representa el $2 \%$ y el $5 \%$ respectivamente del total de los entrevistados. Esto nos confirma el hecho de que muchos gerentes de las Pymes se forman empíricamente y toman decisiones sobre las situaciones que se presentan a diario y no hacen ningún tipo de planeación sobre las políticas de su empresa.

\section{FIGURA 5}

\section{Nivel educativo alcanzado de quien administra el hotel}

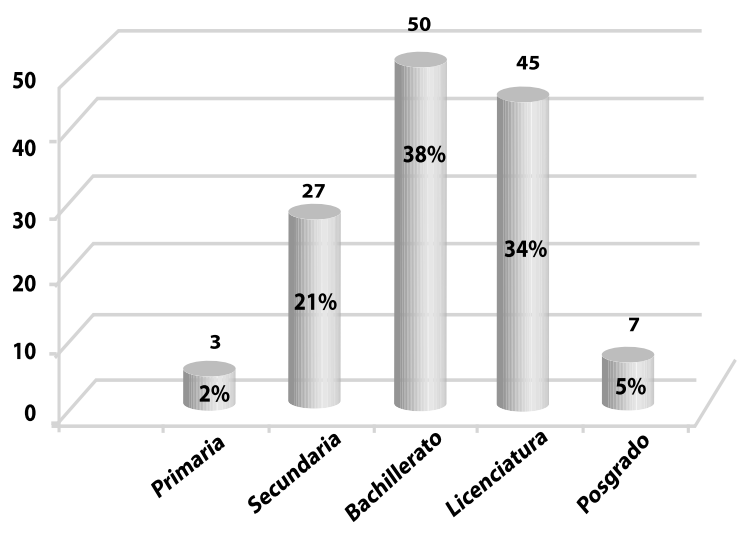

Ampliando este análisis, los resultados de la tabla 1 muestran que de los dueños que administran su hotel, tres alcanzaron como nivel máximo educativo la primaria, 17 secundaria, 33 bachillerato, 18 licenciatura y dos nivel de posgrado. En lo que se refiere a los gerentes que son familiares de los dueños, encontramos que uno obtuvo el nivel de secundaria, cuatro de bachillerato, cinco de licenciatura, dominando ligeramente los de nivel de licenciatura. En lo que respecta a los gerentes que no tienen relación familiar alguna con los dueños del hotel, se encontró que nueve alcanzaron el nivel de secundaria, 13 de bachillerato, 22 de licenciatura y cinco de posgrado. Haciendo notar que estos dos últimos niveles educativos dominan en todas las categorías y demostrando que estos gerentes alcanzaron un nivel educativo más alto que el resto de los demás.

TABLA 1

\section{Nivel educativo alcanzado por tipo de gerente}

\begin{tabular}{|c|c|c|c|c|c|c|}
\hline & 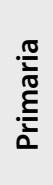 & 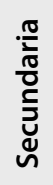 & 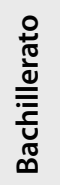 & 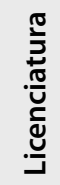 & $\begin{array}{l}\circ \\
\frac{0}{0} \\
\overline{0} \\
0 \\
\circ\end{array}$ & $\begin{array}{l}\overline{0} \\
\text { 。0 }\end{array}$ \\
\hline Dueño & 3 & 17 & 33 & 18 & 2 & 73 \\
\hline Familiar & 0 & 1 & 4 & 5 & 0 & 10 \\
\hline Gerente & 0 & 9 & 13 & 22 & 5 & 49 \\
\hline Total & 3 & 27 & 50 & 45 & 7 & 132 \\
\hline
\end{tabular}

Asimismo, de los dueños que administran un hotel 36 son hombres y 37 mujeres, lo que representa el 50\% para cada uno de los gerentes en esta categoría. De los familiares de los dueños que administran un hotel, nueve son hombres y una es mujer, con lo que se ve claramente que en esta categoría dominan los hombres. En lo que se refiere a los gerentes que no tienen alguna relación familiar con el dueño 38 son hombres y 11 mujeres.

Finalmente, en lo referente a la edad promedio de quien administra un hotel, se encuentra una gran diferencia entre la edad mínima y máxima de un gerente siendo de 28 años la primera y de 66 años la segunda. Se puede decir que entre los grupos de edad de 31 años y 50 años se encuentra la mayoría de los gerentes, esto representa casi el $77 \%$ del total de los gerentes y que hay una minoría entre el grupo de 28 y 30 años, representando el $6 \%$ del total, asimismo hay un grupo importante de gerentes con una edad entre los 51 y 60 años que representan casi el 
17\% del total de esta categoría. Esto indica que predominan los gerentes que se encuentran en el grupo de 31 a 40 años (ver figura 6).

\section{FIGURA 6}

\section{Edad de quien administra el hotel por grupos de edad}

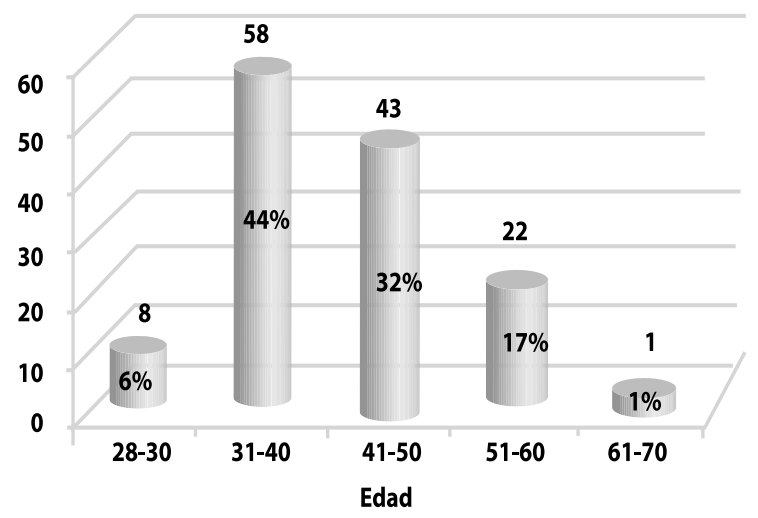

Del nivel características del hotel, los resultados muestran que el número de habitaciones promedio con que cuentan los hoteles varía bastante ya que se encontró un hotel con un máximo de 187 habitaciones y un hotel con un mínimo de 10 habitaciones. Por otro lado, respecto al número de trabajadores promedio que laboran en los hoteles se observa que en los datos obtenidos hay un mínimo de 4 trabajadores y un máximo de 25 trabajadores. En lo referente al número de años promedio de estar operando los hoteles tomados en cuenta para esta investigación, los resultados muestran que existe un máximo de 45 años y un mínimo de 3 años, esto indica que existen muchos hoteles operando desde hace muchos años y que requieren de mucha inversión para su renovación o remodelación, de manera que sean acordes con los requerimientos de la demanda, así como varios hoteles relativamente nuevos.

En lo que concierne al nivel de ocupación habitacional promedio anual en porcentaje del año
2006, los resultados muestran que hubo un mínimo del $45 \%$ y un máximo del 90\%, obteniendo un promedio anual del 72\%. Para el año 2007, los resultados muestran que hubo un mínimo del $48 \%$ y un máximo del 90\%, obteniendo igual que el año anterior un promedio del $72 \%$ de ocupación habitacional anual. En lo que respecta al año 2008, los datos muestran que hubo un mínimo del 48\% y un máximo del 90\%, indicándonos que el promedio anual ocupacional de los hoteles fue del 73\%, manteniéndose casi por igual en los tres años tomados en cuenta.

En cuanto al tipo de turistas que recibe el hotel, los resultados indican que casi todos los hoteles reciben mayoritariamente turistas nacionales (mexicanos) y solo un hotel recibe turistas extranjeros mayoritariamente, sin mencionar la proporción de cada uno, ya que puede variar según la temporada vacacional. Finalmente, el promedio anual de la estancia en días de los turistas de los años 2006, 2007, y 2008 fue de 4 días de acuerdo con los resultados, teniendo un máximo de 5 días y un mínimo de 3 días igualmente para los tres años, no encontrando ninguna variación en la estancia en esos años.

Los resultados de la tercera dimensión "Características empresariales del dueño/gerente", se muestran en la figura 7 e indican que en la variable "Conocimiento de la actividad turística", 64 dueños/gerentes tienen un conocimiento fuerte y muy fuerte de la actividad turística, esto representa el $48 \%$ del total de los entrevistados, 34 consideran que tienen un conocimiento medianamente débil, representando el $26 \%$ y el mismo número de entrevistados consideran que tienen un conocimiento débil de la actividad, estos dos ítems juntos representan el 52\%, lo cual indica que la mayoría de los dueños/gerentes entrevistados tienen una debilidad que es el desconocimiento de la actividad turística, lo que les impide elaborar planes de desarrollo y establecer políticas a corto, mediano y largo plazo. 
FigURA 7

\section{Conocimiento de la actividad turística}

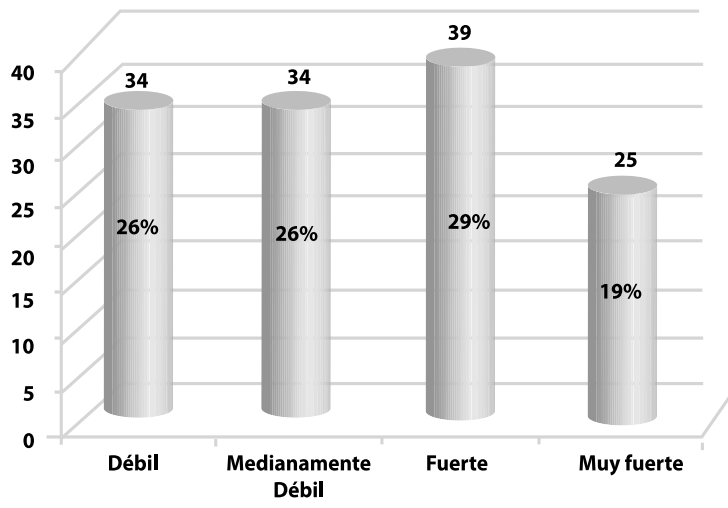

En lo que respecta a la variable "Experiencia en la hotelería", 97 dueños/gerentes indican que tienen una fuerte y muy fuerte experiencia, esto representa el $74 \%$ del total, de los que consideran que tienen una experiencia medianamente débil son 31, y representan el 24\%. Lo anterior indica que a pesar de tener experiencia en lo hotelería, no ha sido suficiente para llevar a su hotel a un crecimiento ya que desconocen qué pasa en el entorno de su hotel. Sobre la variable "Habilidades empresariales", los resultados revelan que 68 entrevistados consideran que tienen entre fuertes y muy fuertes habilidades, representando el 52\% y por otra parte, 53 entrevistados mencionan tener habilidades medianamente débiles, esto representa el $40 \%$, que es una cantidad alta de los que carecen de habilidades empresariales, y 11 empresarios mencionan tener habilidades débiles y muy débiles, esto representa el $8 \%$ del total en esta categoría (ver figura 8).

Sobre la variable "Formación profesional", 49 de los entrevistados consideran que tienen una formación fuerte y muy fuerte, representando el $37 \%$, de los que consideran que tienen una formación medianamente débil representan el
FIGURA 8

\section{Experiencia en la hotelería}

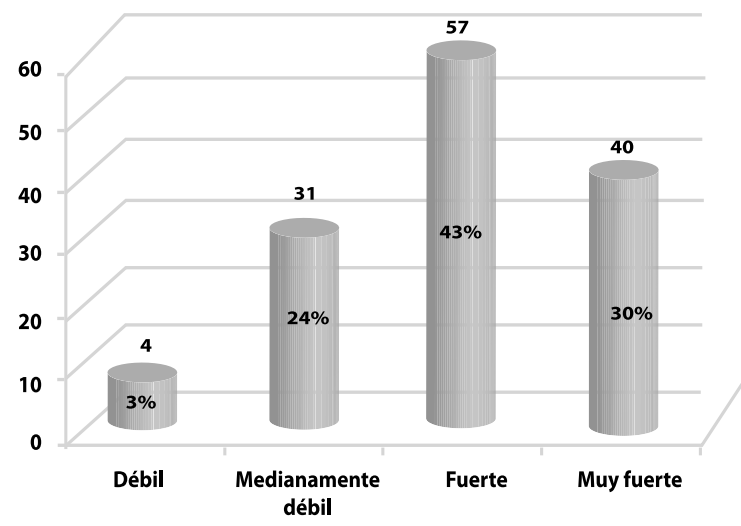

$25 \%$, es decir 33 entrevistados y de los que consideran que tienen una formación profesional débil, que representan el 32\%, son 42 dueños/ gerentes. Como se puede observar, en esta variable predominan los que tienen una formación profesional débil, esto representa una debilidad que muchos gerentes/dueños tienen que superar ya que se ve reflejado en la gestión de su hotel en forma negativa.

En la tabla 2 sobre la variable "Antecedentes familiares empresariales", se observa que 51 de los entrevistados mencionaron que sí tienen antecedentes y 81 que no los tienen, representando el $39 \%$ y $61 \%$ del total respectivamente. Asimismo, de los gerentes que tienen antecedentes 33 son dueños, tres son familiares del dueño y 15 no tienen ninguna relación familiar; de los que no tienen antecedentes, 40 son dueños, siete son familiares del dueño y 34 no tienen ninguna relación familiar. Lo anterior nos indica que predominan los gerentes que no tienen antecedentes familiares empresariales. 
TABLA 2

\section{Antecedentes familiares empresariales por gerente del hotel}

\begin{tabular}{|c|c|c|c|c|c|}
\hline 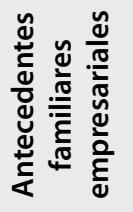 & 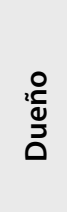 & 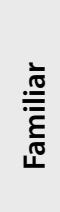 & 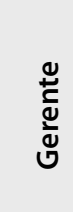 & $\begin{array}{l}\overline{\text { లँ }} \\
\stackrel{0}{\circ}\end{array}$ & 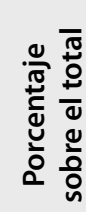 \\
\hline $\mathrm{Si}$ & 33 & 3 & 15 & 51 & $39 \%$ \\
\hline No & 40 & 7 & 34 & 81 & $61 \%$ \\
\hline
\end{tabular}

Ampliando los resultados anteriores, en la tabla 3 se muestra que de los gerentes que tienen antecedentes familiares empresariales 33 son hombres y 18 mujeres y de los que no tienen antecedentes 50 son hombres y 31 mujeres. Predominando en ambos casos los hombres.

TABLA 3

\section{Sexo de quien administra el hotel y antecedentes familiares empresariales}

\begin{tabular}{|c|cc|c|}
\hline $\begin{array}{c}\text { Antecedentes } \\
\text { familiares } \\
\text { empresariales }\end{array}$ & \multicolumn{2}{|c|}{$\begin{array}{c}\text { Sexo } \\
\text { de quien } \\
\text { administra }\end{array}$} & Total \\
\hline Hombre & Mujer & \\
\hline Si & 33 & 18 & 51 \\
\hline No & 50 & 31 & 81 \\
\hline Total & 83 & 49 & 132 \\
\hline
\end{tabular}

Finalmente, de los resultados obtenidos, 33 hoteles manifestaron haber crecido en esos tres últimos años lo que representa un 25\% del total de hoteles participantes mientras que 99 manifestaron no haber crecido, es decir un $75 \%$ del total. Ampliando la información, se encontró que de los dueños que administran un hotel 12 sí crecieron y 61 no lo hicieron, mientras que de los hoteles que son administrados por algún familiar del dueño, cuatro si crecieron y seis no lo hicieron y de los hoteles administrados por un gerente sin ninguna relación con el dueño, crecieron 17 y 32 no lo hicieron. Como se puede observar en los datos anteriores, de los 33 hoteles que lograron crecer, solo un $36 \%$ son administrados por los propios dueños, mientras que las Pymest que tuvieron más éxito en su crecimiento fueron las administradas por gerentes que no tienen relación con el dueño, y que representan un 52\% del total de los que sí crecieron, situación que puede encontrar su explicación en el hecho de que estos últimos son quienes tienen un mayor nivel de estudios (ver tabla 4).

TABLA 4

\section{Administración del hotel y crecimiento en los últimos años}

\begin{tabular}{|c|cc|c|}
\hline $\begin{array}{c}\text { Administración del } \\
\text { hotel }\end{array}$ & \multicolumn{2}{|c|}{$\begin{array}{c}\text { Ha crecido en los } \\
\text { últimos años? }\end{array}$} & Total \\
\hline Si & No & \\
\hline Dueño & 12 & 61 & 73 \\
\hline Familiar & 4 & 6 & 10 \\
\hline Gerente & 17 & 32 & 49 \\
\hline Total & 33 & 99 & 132 \\
\hline
\end{tabular}

\section{CONCLUSIONES}

Derivado de los resultados anteriores las conclusiones más relevantes en esta investigación son las siguientes:

Las Pymest en Acapulco que se identificaron con mayor crecimiento en el periodo considerado por el estudio, fueron las que son administradas 
por gerentes que no son dueños ni familiares de éstos y que además presentan un mayor nivel educativo, así como el hecho de contar con antecedentes familiares empresariales, características que concuerdan con lo señalado por Haber y Reichel (2005 y 2007) en cuanto a la importancia del conocimiento de la actividad turística y de las características empresariales de quien administra una Pymest, en este caso un hotel.

El dinamismo de la actividad turística y su entorno son sin duda factores que si son aprovechados, pueden propiciar el crecimiento de las Pymest, para ello, es necesario que el dueño/ gerente cuente con el conocimiento de la actividad pero también con la orientación empresarial que le permita desarrollar las habilidades para administrar una Pymest.

Independientemente de que esta investigación se basó en la percepción y opinión de los participantes -lo cual limita la generalización de los resultados-, su aportación académica se considera significativa, en el sentido de que sienta las bases para generar varias líneas de investigación sobre las Pymes turísticas en México, en sectores como: restaurantes, agencias de viajes, centros nocturnos, parque recreativos, entre otros, que además son complementos importantes de la actividad turística de un destino.

Finalmente, este tipo de investigaciones permitirá implementar políticas estratégicas y acciones donde se involucre al gobierno a nivel Federal, Estatal y Municipal, y a los propios empresarios a fin de favorecer el crecimiento de las Pymest a largo plazo.

\section{REFERENCIAS}

Ateljevic, J. (2007). Small tourism and management practices in New Zeland. The centre stage macro region. Case study. Tourism Management, 28, 307-316.

Bates, T. (2005). Analysis of young, small firms that have closed: Delineating successful from unsuccessful closures. Journal of Business Venturing, 20, 343-358.

Birch, D. (1987). Job creation in America: How Our Smallest Companies Put the Most People to Work. New York: The Free Press.

Calvo, A. y López, V. (2004). Recursos estratégicos intangibles y rendimiento: un

análisis empírico. Revista Europea de Dirección y Economía de la Empresa, 13(3), 49-64.

Capelleras, J. y Kantis, D. (2009). Nuevas empresas en América Latina: factores que favorecen su rápido crecimiento. Editores: Universidad Autónoma de Barcelona y Universidad Nacional de General Sarmiento.

Chen, T. (2009) ."Performance measurement of an enterprise and business units with an application to a Taiwanese hotel chain". International Journal of Hospitality Management, 28, (3), 415-422.

Claver, E., Andrew, R. y Quer, D. (2006) Growth strategies in the Spanish hotel sector: determining factors. International Journal of Contemporary Hospitality Management, 18(3), 188-205.

Cooper, A., Gimeno-Gazcon, F. (1994) Initial human capital and financial capital as predictors of new venture performance. Journal Business Venturing. 9 (5), pp. 371 395.

Covin, J.G. y Slevin, D.P. (1989). Strategic management of small firms in hostile and benign environments. Strategic Management Journal,10, 75-87.

Davidson, P. y Wiklund, J. (1999) Theoretical and Methodological Issues in the Study of Firm Growth. Jonkoping International Business School, Working Paper Series: 99-6.

Davidson, P. (2002) Continued entrepreneurship: ability, need and opportunity as determinants of small firm growth. Sweden: University, Business School UMEA

Dobbs, M. y Hamilton, R.T. (2007). Small business growth: recent evidence and new directions. International Journal of Entrepreneurial Behaviour \& Research,13 ( 5), pp. 296-322. 
Druker, P. (1985) Entrepreneurship and innovation. New York: Harper and Row, Publishers.

Haber, S. y Reichel, A. (2005) Identifying performance measures of small ventures. The case of Tourism industry. Journal of Small Business Management. 43(3), pp. 257286.

Haber, S. y Reichel, A. (2007) The cumulative nature of the entrepreneurial Process: The contribution of human capital, planning and environment resources to small venture performance. Journal of Business Venturing, 22, pp. 119-145.

Haktanir, M. y Harris, P. (2005). Performance measurement practice in an independent hotel context: a case study approach. International Journal of Contemporary Hospitality Management, 17( 1), pp. 39-50.

Instituto Nacional de Estadística, Geografía e Informática (INEGI), México (2009).

Jogaratnan, G., Mclearly, K. y Mena, M. (2005) An analysis of hospitality and tourism research: institutional contribution. Journal of Hospitality and Tourism Research, 29 (3), 356-371.

Mazzarol, T. (2003). A Model of Small Business HR Growth Management. International Journal of Entrepreneurship Behaviour \& Research, 9(1), 27-49.

Mill, R.C. y Morrison, A. (1992) The Tourist System. Eds. Kendall/Hunt Publishing Company $4^{\text {Th }}$ edition.

Miner, J. (1996) Entrepreneurs, high growth entrepreneurs and managers: contrasting and overlapping motivational patterns. Journal of Business Venturing, 5, 221234.

Monfort, V. (2000) Competitividad y factores críticos de éxito en la hotelería de Litoral, experiencia de los destinos turísticos Benidorm y Peñíscola. FITUR 2000, Madrid.

Organización Mundial de Turismo (2008) Noticias OMT, año XXII, número 2.

Palomo, M.A. (2007) La gestión de procesos y el desempeño competitivo de las pymes, Ingenierías, abril-junio, $X, 35$.

Pechlaner, H. y Matthias, F. (2004) Toward new skill requirements for destination

organizations: an exploratory study. Tourism Analysis, 7(1), pp. 43-53.
Pechlaner, H., Raich, F., Zehrer, A. y Peters, M. (2004) Growth perceptions of small and medium-sized enterprises (SME's) - The case of South Tyrol, Italia. Tourism Review, 59 (4), pp 7-13.

Phillips, P. (1999) Hotel performance and competitive advantage: a contingency approach. International Journal of Contemporary Hospitality Management, 11/7, pp. 356-365.

Rabetino, R. (2005) Factores Determinantes del Crecimiento en el Empleo de las Nuevas Empresas en Latinoamérica. Trabajo de Investigación, Universidad Autónoma de Barcelona.

Rubio, A. y Aragón, A. (2008) Recursos estratégicos en la Pyme: una aproximación empírica. Revista Europea de Dirección y Economía de la Empresa,17 ( 1), pp. 103-126.

Schutjens, V. y Wever, E. (2000) Determinants of new firm success. Regional Science. 79, 135-159.

Smallbone, D., Leigh, R. y North, D. (1995) The characteristics and strategies of high growth SMEs. International Journal of Entrepreneurial Behaviour \& Research. 1( 3), pp. 44-62.

Smith, S. (2006) How big, How many? Enterprise size distribution in tourism and other industries. Journal of Travel Research, 45, 53-58.

Secretaría de Fomento Turístico del Estado de Guerrero (Sefotur), (2009).

Secretaría de Turismo Federal (Sectur), México (2009).

Storey, D. (1994) Understanding the Small Business Sector. London: Routledge.

Turok, I. (1991) Which small firms growth? eN L.G. Davies \& A.A. Gibb eds. Recent Research in Entrepreneurship, Avebury, Aldershot, England, pp. 29-44.

Weiermair, K. (2001) Theoretical Foundations or Considerations regarding the Growth of Tourism Enterprises. Tourism Review, 3+4.

Wiklund, J. (1998) Small Firm Growth and Performance: Entrepreneurship and Beyond (Tesis Doctoral), Jonkoping International Business School.

Wiklund, J. y Sheperd, D. (2003) Aspirin for, and Achieving Growth: The Moderating Role of Resources and Opportunities. Journal of Management Studies. 40 (8), 1919-1941. 
RNA Revista Nacional de Administración

Wiklund, J., Davidson, P. y Delmar, F. (2003) What Do they Think and Feel about Growth? An expectancy-Value Approach to Small Business Manager's Attitudes Toward Growth. Entrepreneurship Theory and Practice. Spring, pp. 247-270.

Wiklund, J. y Sheperd, D. (2005) Entrepreneurial orientation and small business performance: a configuration approach. Journal of Business Venturing. 20, pp. 71-91.

Recibido: 17 de enero de 2011 Aceptado: 23 de febrero de 2011 